\title{
Potential dangerous object detection on railway ballast using digital image processing
}

\author{
P. L. Mazzeo, E. Stella, M. Nitti \& A. Distante \\ Istituto di studi sui sistemi intelligenti per l'automazione, \\ Consiglio Nazionale delle Ricerche, Italy
}

\begin{abstract}
The correct assessment of the condition of a railroad requires the consideration of different factors. Some factors, such as the condition of the ties, can be measured by inspecting features visible from the surface of the railway. Other factors include the condition of the ballast; it is important to recognize the critical situation in which any foreign object can be present on the ballast. These kinds of objects could be cans, pieces of sheet and everything over a well determined dimension. Extensive human resources are currently applied to the problem of evaluating railroad health. The proposed visual inspection system uses images acquired from a digital line scan camera installed under a train.

Here we focus on the problem of foreign object detection in the railway maintenance context. To obtain this aim we train a Multilayer Perceptron Network (MLPN) with the edge histogram of the ballast patches manually extracted from the acquired digital image sequence. The general performances of the system, in terms of speed and detection rate, are mainly influenced by the adopted features for representing images and by their number. By this inspection system it is possible to aid the personnel in railway safety issues because a high detection rate percentage has been obtained. We show the adopted techniques by using images acquired in real experimental conditions.
\end{abstract}

Keywords: obstacle detection, ballast inspection, neural networks.

\section{Introduction}

Inspection of the rail state is one of the basic tasks in railway maintenance. In the last few years a large number of methods have been proposed by the computer vision community for facing the problem of visual inspection $[1,2]$. The 
problem is a particular instance of the most general question of detecting objects in digital images as faces [3], pedestrian [4], balls [5], just for citing a few examples. Recently, such methods have been successfully applied for railway inspection and monitoring [6, 7]. In fact, the growing of the high-speed traffic on the rail tracks demands the development of sophisticated real-time visual inspection systems. The railroad network must be continuously maintained to assure the safe and timely delivery of freight and passengers. This maintenance effort is complicated by the fact that accurate assessments of track condition are difficult and expensive to obtain. In many cases, efficient data collection tools exist, but human experts are required to analyze the data and identify sections of track that require maintenance. With thousands of miles of track, this task is very often impractical. In this paper, we apply pattern recognition techniques in order to automate the analysis of railroad data. Normally the maintenance of the railway plane is done by trained personnel who periodically observe the images recorded by a TV camera installed on a diagnostic coach. This manual inspection is lengthy, laborious and potentially hazardous and the results are strictly dependent on the capability of the observer to catch possible anomalies and recognize critical situations.

A considerable body of work has been published on the problem of applying recent technology advances to aid in the classification and management of railroad systems. Computers have been used to help automate the collection and analysis of railroad data as early as 1967 [9]. Sensing systems capable of collecting various track geometry data have been developed and applied. Systems capable of recording track geometry and and strength have been developed for railroad cars [10] and high-rail vehicles [11]. Computer vision systems have been applied to monitor rail wear [12], measure rail width [13], and produce a database of continually scanned visual images [14]. These systems facilitate the automated collection of large amounts of railroad data. However, the data must be parsed and analyzed by a human expert before it can be used to make maintenance decisions.

Similar advances have been made in the context of detecting of sleepers' anomalies, as well as missing fastening elements, is an important task that an efficient inspection tool should supply. As described in our previous work [6] the Wavelet Transform has been successfully applied in railway context for the recognition of fastening elements. In other works $[8,15]$ this fastening element are recognized by using Independent Component Analysis (ICA) and Support Vector Machine (SVM). This kind of detection problem can be regarded as the problem of detecting flat objects from 2-D intensity image. Usually, such problems have been approached by using algorithms of edge detection, border following, thinning, straight line extraction, active contour (snake) following [16]. In this work we focalize on the ballast recognition in order to catch the possible presence of every potential dangerous foreign object (cans, piece of sheet etc. plastic bottles) that do not belong to railroad structure. 
There are few works that focus on the measurement of ballast and subgrade condition. In these works the primary tool for non-destructive measurement of subsurface condition is ground penetrating radar (GPR). GPR has been used to identify fouled ballast and trapped water on an experimental railroad test bed [17]. In [18] GPR was found particularly useful for locating drainage problems and identifying areas of similar substructure condition. Other efforts have been successfully combined GPR with infrared imaging systems to detect subsurface defects in railroad track beds [19]. However no works have been found on ballast inspection in order to recognize potential dangerous object that not belong to railroad bed. The main idea of this work is to find a non-invasive technique that use the images captured by a TV Camera. To reach this aim it has been found a technique based on the edge-histogram combined with a neural classifier (Multi Layer Perceptron). The main idea behind this technique is that any foreign object that does not belong to railroad ballast has a well-defined structure. In this way we can characterize two object classes: the ballast, and anything that is not ballast. The texture classification techniques are not used because the ballast is not a texture. It is not a function of the spatial variation in pixel intensities (gray values) which can characterize the ballast distribution. Furthermore, the ballast has very different pixel intensity (a great variability of gray level luminosity) and the dimension of each stone is strongly variable. In this work we present a collection of cases in which our technique works as ballast detector and we present a table where a detection rate percentage gives a measure of the robustness and reliability. This paper is organized as follows: In section 2 an overview of the implemented system is given. In section 3 we briefly describe of the pre-processing used technique. The MLP classifier is succinctly introduced in section 4. Finally experimental results are given in section 5 .

\section{System overview}

As introduced in the previous section, we have realized a vision architecture that is able to detect the ballast region in the digital image acquired. This system identifying the ballast region can account the critical situation in which any potential dangerous object is presented on the ballast surface. This type of anomaly is very dangerous because when it occurs can obstacle the normal train traffic, generating consequences for safety issues. In truth the potential dangerous objects can lie on the sleepers but in this case they will be treated in different way.

The proposed system architecture is based on data classification by using a supervised learning scheme. The logical scheme of the whole system's processing block is showed in figures 1 and 2 . The system is composed by 3 main blocks:

- Prediction Algorithm Block (PAB);

- $\quad$ Rail Detection and Tracking Block (RD \& TB);

- Ballast Identification Block (BIB). 
PAB extracts from an input video sequence the images areas candidate to contain the sleepers. This block takes advantage of the distance between two consecutive sleepers that is a priori known distance.

Rail Detection and Tracking Block determines the rail position respect than acquired images and tracks it in the video sequence. BIB examines the areas where is the ballast and determines the situations in which potential dangerous objects are presented.

By this blocks the systems is able to identify the sleepers (PAB) and it enables the Ballast Identification Block (BIB) only in that areas where is present the ballast. Observes that without the PAB the system could classify the sleeper regions as foreign objects because it does not belong to the ballast class. This algorithm jointly to Rail Detection and Tracking Block (RD \& TB) works to determine the region candidate to contain the ballast. PAB [20] uses the rail position (determined by RD \& TB) for calculating the distance between two consecutive sleepers in order to predict the position of the image areas candidate to contain the ballast patches. In figure $\mathrm{Y}$ is exploded the Ballast Identification Block (BIB) and all its functional parts are shown.

This Block is subdivided in two functional sub-blocks:

- $\quad$ Edge Detection \& Histogram Block (ED \& HB);

- Multi Layer Perceptron Network Block (MLPNB).

The first block performances a pre-processing on the input patches in order to extract the features which characterize the images. The extracted features are the input coefficients of the MLPN contained in the second block.

The reduction of the input space dimension performed by the ED \& HB is the first step to successfully speed up the whole classification process. This target can be reached by using a feature extraction algorithm which should be able to store all the significant information about the input patterns in a set with few important elements. To achieve these requirements we have used the edgehistogram pre-processing technique as shown in figure 2. Used approach allowing to capture the object structure that distinguish the potential dangerous object between the ballast.

The output of ED \& HB is a coefficient features vector that is the input of a MLPNB. More details are equipped in the next sections. Finally BIB produces a Pass/Alarm signal which is online displayed. In case of alarm (i.e. potential dangerous object detected on the ballast) BIB records the coordinate into a log file.

\section{Pre-processing}

As delineated in previous section the implemented system extracts from the candidate patches the edge histogram. This method captures non-directional edges as well as any directional ones. For the edge extraction step we have used the Sobel edge detector mask in the horizontal and vertical direction. 


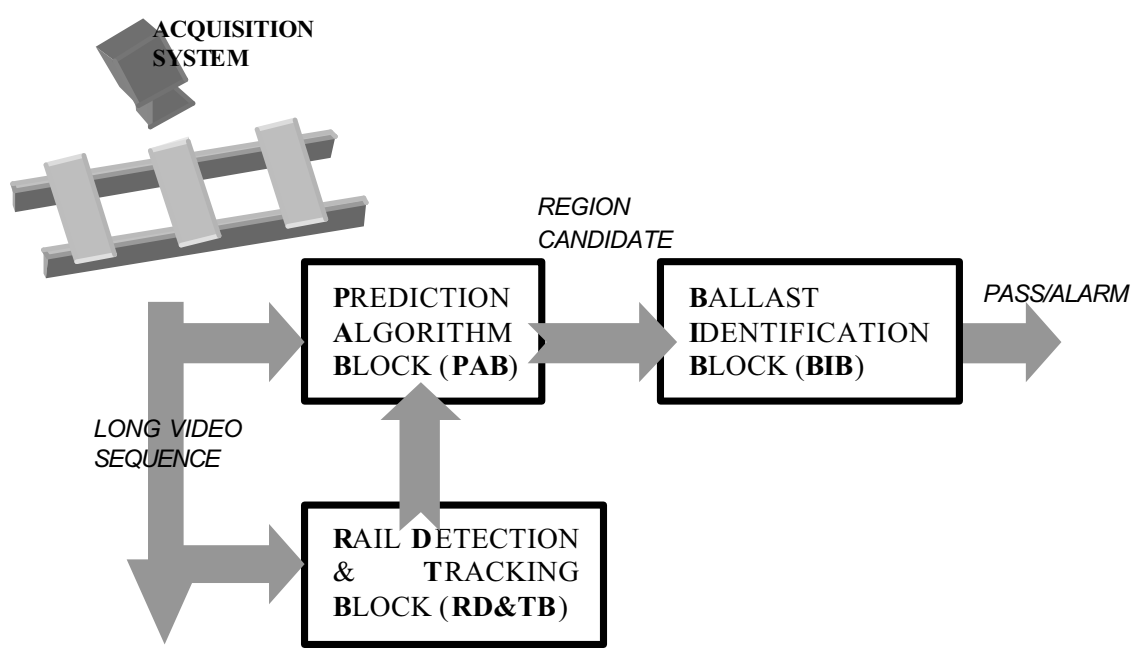

Figure 1: $\quad$ System architecture.

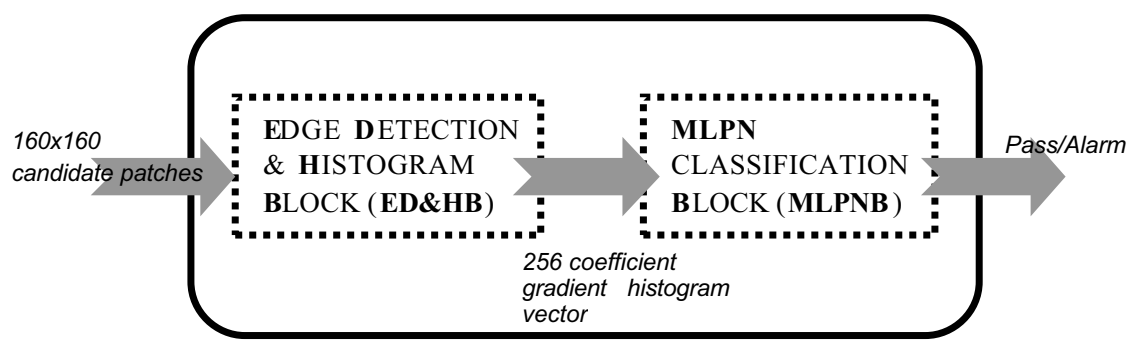

Figure 2: Ballast identification block.

After that we have employed these edge image results to find the approximate absolute gradient magnitude at each point in an input grayscale image:

$$
|\mathrm{G}|=|\mathrm{Gx}|+|\mathrm{Gy}|
$$

where Gx estimating the gradient in the $\mathrm{x}$-direction (columns) and the Gy estimating the gradient in the y-direction (rows).

Finally we calculate the edge histogram on the gradient image G, based on 256 bins that is the number of the grey level images.

Figures 4 and 5 represent the edge histogram of the two image patches that are shown in figure 3. 

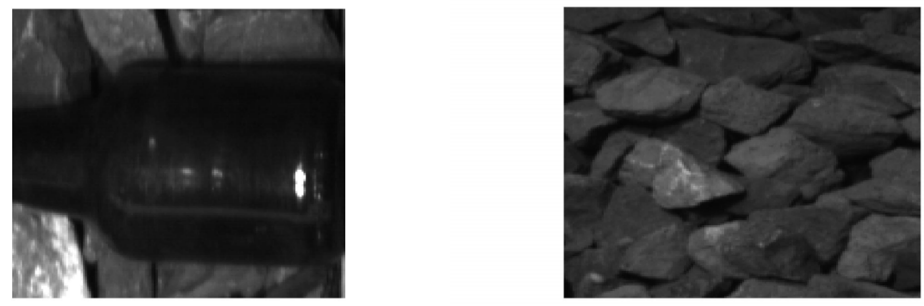

Figure 3: Examples of 160x160 pixels patches: foreign object (left) and ballast (right).
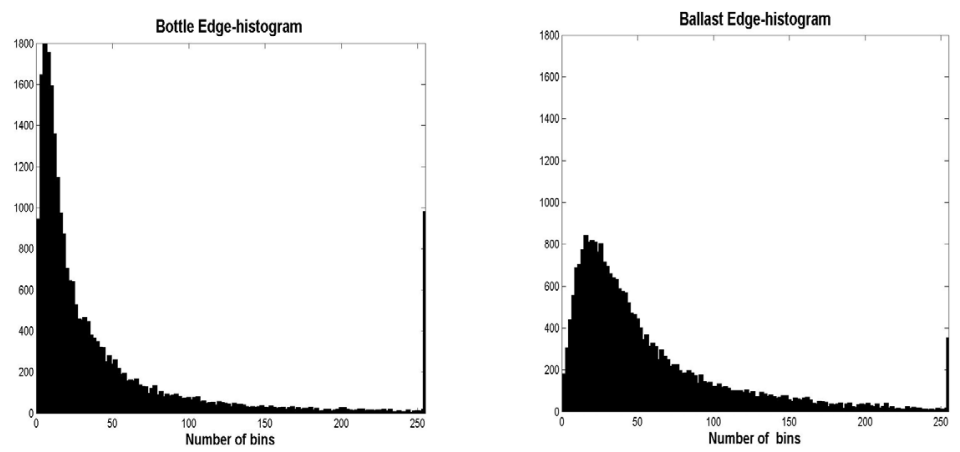

Figure 4: Ballast edge-histogram (right side), dangerous object edge-histogram (left side).
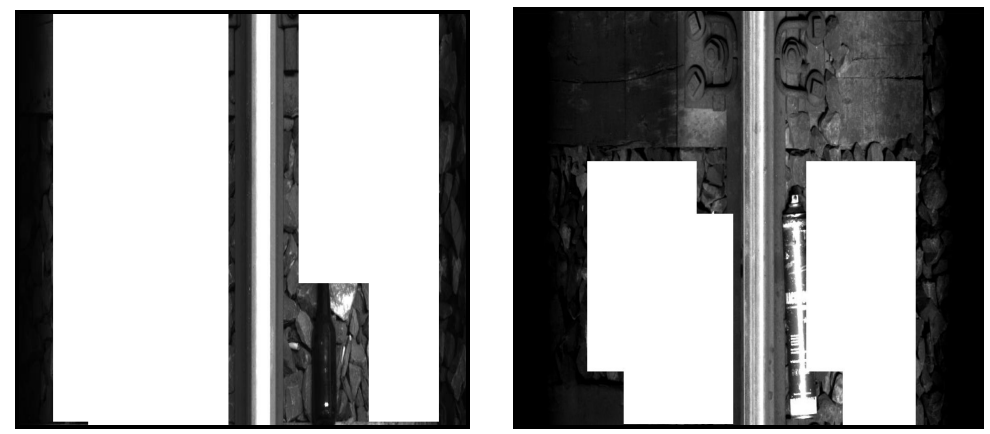

Figure 5: Examples of the system output image. 


\section{Classification}

The classification process has been implemented by using artificial neural networks. Neural computing is particularly successful at extracting data from images. In our particular context of object detection neural-based methods have a key advantage over geometry-based methods because they do not require a geometric model for the object representation. In our detecting system a Multilayer Perceptron trained by Back-Propagation algorithm has been used (MLPN). This architecture consists of three-layers of neurons (input, hidden and output layer). The input layer has a number of neurons equal to the number of image features extracted in the pre-processing step.

The output layer has only one unit which generates an output ranging from 0 to 1 indicating a measure of confidence on the presence of the object to detect in the current image patch. As activation function for both hidden and output units we have used the log-sigmoidal function that generated output between 0 and 1 . Error Back Propagation algorithm with an adaptive learning rate has been used to solve the weights of the MLPN [21].

\section{Experimental results}

The images of the rail have been obtained by a line scan camera DALSA PIRANHA 2 with 1024 pixels of resolution (maximum line rate of $67 \mathrm{kLine} / \mathrm{s}$ ) with the transmission protocol Cameralink, installed under a diagnostic train during its maintenance route. Futhermore we have used the frame grabber PCCAMLINK (Imaging Technology CORECO). In order to reduce the effects of variable natural lighting conditions, an appropriate illumination setup equipped with six OSRAM 41850 FL light sources has been installed too. In this way the system should be robust against changes in the natural illumination. Moreover, in order to synchronize data acquisition, a trigger is sent to the TV camera by the wheel encoder.

The spatial resolution of the trigger is $3 \mathrm{~mm}$. A pixel resolution of $1 \times 1 \mathrm{~mm}^{2}$ can be obtained choosing a TV camera with focal length of $12 \mathrm{~mm}$. The integration time of the TV camera has been properly set in order to acquire images at maximum speed of $241 \mathrm{~km} / \mathrm{h}$ choosing the spatial resolution of $1 \mathrm{~mm}$. A long video sequence of a rail network has been acquired in order to experiment the proposed visual-based inspection system. Firstly, a number of sample images has been extracted from the sequence to create the training set for the neural classifiers. The remaining video sequence has been used to test the performance in term of detection rate e computational velocity of the developed inspection system.

Positive and negative examples of the ballast patches have been manually extracted from the sequence training images (see figure 3 right side). In the experiment stage we have used a crescent patch dimension in order to determine the best compromise between the ballast identification process and the consequent potential dangerous objects identification. Anyway maximun 
dimension of the example patches consist of a 160x160 pixels subwindow (see figure 3).

This training set contains 520 positive examples (ballast) and 183 negative examples (foreign objects). The used Multi Layer Perceptron is composed by 256 input layer neurons 30 hidden layer ones and one output neuron. The same neural classifier has been trained by the edge histogram as explained in section 3 in this way the input space dimension has been reduced to 256 feature coefficients. The neural classifiers have been trained on the same number examples training sets. In order to evaluate the generalization ability of the classifier a preliminary test has been carried out on a validation set.

This set contains 800 positive examples (ballast patches) and 300 negative ones (foreign object patches). In table 1 the results of that test are shown. In the first column of Table 1 the used patch dimension are listed. Detection rates are given in terms of true positive (TP) and true negative (TN). From this table can be found out that the different trained classifiers perform almost well in all the considered cases of the patches dimensions. However the best result obtained corresponds to the last row in the table 1.

Table 1: $\quad$ System detection rate on a validation set.

\begin{tabular}{ccc}
\hline \hline $\begin{array}{c}\text { Patch } \\
\text { dimensions }\end{array}$ & $\begin{array}{c}\text { True Positive } \\
(\%)\end{array}$ & $\begin{array}{c}\text { True Negative } \\
(\%)\end{array}$ \\
\hline $40 \times 40$ & 87.98 & 94.55 \\
$80 \times 80$ & 91.92 & 93.95 \\
$160 \times 160$ & 95.38 & 97.56 \\
\hline \hline
\end{tabular}

For this reason, the patch dimension of 160x160 pixels have been chosen for the second phase of testing experiment. In that experimental phase a long video sequence of rail has been inspected by the implemented vision system. The system inspects only the image areas that are candidates to contain the ballast.

Figure 5 shows how the system works. The white regions are the patches that system identifies like ballast and the reader could be see that the foreign object are correctly identified.

\section{Conclusion and future work}

This paper has proposed a prototype able to detect autonomously potential dangerous object on the ballast region. The periodical inspection of railway infrastructures is very important to prevent dangerous situations. The developed system can be used to detect probable dangerous object on the ballast region in railway infrastructure.

The inspection system uses images acquired by a digital line scan camera installed under the train. Neural classifier has been trained to recognize ballast patches in railway track. Firstly the images are pre-processed by using edge- 
histogram method. Then the obtained detecting system has been tested on a validation set to establish the best patch dimension which give us the best performance. After that, a long sequence of tests on real images has been examined. The system correctly detects the ballast region as well as the presence of foreign object. By our inspection system is possible to aid the personnel in the railway safety issue because high percentages of detection rate has been obtained showing a high reliability and robustness.

\section{References}

[1] O. Silven, M. Niskanen, H. Kauppinen. Wood inspection withnonsupervised clustering. Machine Vision and Applications (2003) Vol. 13 No.5-6: 275-285.

[2] Y. Zhang, Z. Zhang, J. Zhang. Deformation visual of industrial parts with image sequence. Machine Vision and Applications (2004) Vol. 15 No.3 115-120.

[3] K. Sung and T. Poggio. Example-based Learning for View-based Human Face Detection. Artificial Intelligence Laboratory. Massachusetts Institute of Technology, Cambridge, MA. 1994. A.I. Memo No. 1521.

[4] C. Papageorgiou and T. Evgeniou and T. Poggio. A trainable pedestrian detection system. Proceedings of Intelligent Vehicles.Stuttgart, Germany, October, 1998.

[5] N. Ancona and G. Cicirelli and E. Stella and A. Distante. Ball detection in static images with Support Vector Machines for classification. Image and Vision Computing. Elsevier 2003. 21(8) 675-692.

[6] P.L. Mazzeo, M. Nitti, E. Stella and A. Distante, "Visual recognition of fastening bolts for railroad maintenance", in Pattern Recognition Letters, vol. 25 no. 6, pp. 669-677 2004.

[7] M. Nitti, P.L. Mazzeo, E. Stella and A. Distante "Real time Position Estimation of overhead line in railway application" in Computers in Railway IX, WIT Press, pp. 835-843, 2004.

[8] Mazzeo P.L., Ancona N., Stella E. and Distante A.: Visual Recognition of hexagonal headed bolts by comparing ICA to Wavelets. Proceedings of the IEEE Inter. Symposium on Intelligent. Control-Houston(2003) 636-641 .

[9] Mechanized automed, computerized, Railway and Track Structures, ,March (1971) 18-21.

[10] W. Ebersohn and E.T. Seling, Use of geometry measurement for maintenance planning. Transportation Research Record No. 1470, Railroad Research Issues (1994) 84-92.

[11] A.M. Zarembski and W.T. MacCarthy, Development of nonconvetional tie and track structure inspection systems. Transportation Research Record No. 1489, Railroad Trasportation Research (1994) 26--32.

[12] D.L. Magnus, Non contact technology for track speed rail measurament (ORIAN), Proc. of the SPIE Nondestructive Evaluation of Aging Railroads, 2458 (1995) 45-51. 
[13] R.B. Ryabichenko, S.B. Popov, and O.S. Smoleva, CCD photonic systems for rail width measurement, Proc. of the SPIE Photonics for Transportation, 3901 (1999) 37--44.

[14] H. Sasama, Maintenance of railway facilities by continuously scanned image inspection, Japanese Railway Engineering, 27 (1994) 1--5.

[15] Mazzeo P.L., Ancona N., Stella E. and Distante A.: Fastening Bolts recognition in Railway images by Independent Component Analysis. Proc. 3rd Visualization, Imaging and Image ProcessingBenalmadena(2003) 668-673.

[16] Andrade-Cetto J. and Kak Avinash C.: Object Recognition. Wiley Encyclopedia of Electrical Engineering. vol. Sup. 1 (2000) 449 - 470.

[17] G. Gallagher, Q. Leiper, M. Clark and M. Forde, Ballast evaluation using ground penetrating radar, Railway Gazette International, February (2000), 101--102.

[18] T.R. Sussmann, F.J. Heyns and E.T. Seling, Characterization of track substructure performance, Recent Advances in the Characterization of Pavement Geomaterials, Geotechnical Special Publication, American Society of Civil Engineers (1999), 37-48.

[19] G.J. Weil, Non-destructive, remote sensing technologies for locating subsurface anomalies on railroad track beds, in Proc. of he SPIE, Nondestructive Evaluation of Aging Railroads 2458(1995), 74--81.

[20] P.L. Mazzeo, E. Stella, M. Nitti, A. Distante, Visual Recognition of fastening bolt in railway maintenance context by using wavelet transform, ICGST International Journal on Graphics Vision and Image Processing Special Issue on Wavelets, May 2005, 27- -34.

[21] M. Bishop, Neural Networks for Pattern Recognition, New York 1999, $220-313$. 